\title{
AMCoR
}

Asahikawa Medical University Repository http://amcor.asahikawa-med.ac.jp/

Geriatrics and Gerontology International (2016.10) 16(10):1173-1174.

Bullous pemphigoid and percutaneous endoscopic gastrostomy

Tsukasa Nozu, Toshikatsu Okumura 
1 Bullous pemphigoid and percutaneous endoscopic gastrostomy

2

3 Tsukasa Nozu ${ }^{1}$, Toshikatsu Okumura ${ }^{2}$

$4 \quad{ }^{1}$ Department of Regional Medicine and Education, Asahikawa Medical

5 University, Midorigaoka Higashi 2-1-1-1, Asahikawa, 078-8510, Japan

62 Department of General Medicine, Asahikawa Medical University,

7 Midorigaoka Higashi 2-1-1-1, Asahikawa, 078-8510, Japan

8

9 Author contribution:

10 Tsukasa Nozu drafted the manuscript, revised the article, and approved the

11 final version of the manuscript. Toshikatsu Okumura critically revised the

12 manuscript, and approved the final version of the manuscript.

14 Running title: Bullous pemphigoid and PEG

16 Key words: Bullous pemphigoid, Institutionalized patients, Percutaneous

17 endoscopic gastrostomy, Risk factor, Tube feeding

18

19 Correspondence to: Tsukasa Nozu, MD, PhD, FACP, FJSIM

20 Department of Regional Medicine and Education, Asahikawa Medical

21 University, Midorigaoka Higashi 2-1-1-1, Asahikawa, 078-8510, JAPAN

$22 \mathrm{Ph} ;+81-166-68-2844$

$23 \quad$ Fax; +81-166-68-2846

24 e-mail; tnozu@sea.plala.or.jp 
Dear Editor

Bullous pemphigoid (BP) is an autoimmune subepidermal blistering disorder with autoantibodies directed against BP180 and BP230, mainly occurring in the elderly population. ${ }^{1}$ It is a rare disease among the general population and the incidences vary between 12.1 and 66 per 1 million people per year in European countries. However, it rises to 150 - 330 in people older than 80 years, ${ }^{1}$ and moreover, it was reported to be much higher among elderly in nursing homes, i.e. 4.8 per 100 person per year. ${ }^{2}$ The risk of death for BP patients was two to six times greater than that of age and sex-matched general population. ${ }^{1,3}$ Since management of the institutionalized elders is principally performed by physicians, the diagnosis and treatment of BP are thought to be an important mission for not only dermatologists but also physicians.

Several risk factors for BP such as skin trauma, surgical procedures, neurological disorders, etc. have been reported. ${ }^{3-5}$ Regarding surgical procedures, ostomy surgery such as colostomy or urostomy is known to induce localized BP around stoma. ${ }^{6}$ Since disabled elderly patients undergo percutaneous endoscopic gastrostomy (PEG) frequently, PEG is also presumed to be a risk factor for BP. In this context, we previously performed the retrospective survey with 36 hospitalized elderly patients, aged 64 to 101 years, (5 BP and 31 non $\mathrm{BP}$ cases) staying more than 2 years in order to clarify this issue and published the paper in $2010 .{ }^{7}$ In that survey, all the BP cases developed generalized BP and underwent enteral feeding by PEG or nasogastric (NG) tube. In the univariate analysis, PEG and tube feeding (by 
PEG or NG tube) were associated with BP. The multivariate logistic and Cox proportional hazard regression analysis were performed with age, sex and PEG or tube feeding, demonstrating PEG was independently associated with BP.

As mentioned before, neurological diseases such as Parkinson's disease, dementia, etc. were known to be significant risk for BP. ${ }^{4}$ Besides, bedridden condition was also reported to be an independent risk factor. ${ }^{4}$ In this context, it is reasonable to think that the one of the possible reasons for the link between $\mathrm{BP}$ and neurological disorders can be due to the increased frequency of undergoing PEG in the disabled patients. However, none of the studies have been performed to evaluate a risk factor for BP among the patients with neurological disorders with focusing on PEG.

$\mathrm{BP}$ associated with ostomy surgery is known to be localized but not generalized BP, suggesting that the lesions may be directly related to the skin damage by surgery. The damage to dermo-epidermal junction with subsequent antigen exposure and activation of the immune system leading to the production of autoantibodies might be involved with this association. ${ }^{8}$ However, in our study, all BP cases with PEG were generalized type, suggesting that other factors may contribute to develop the lesions in addition to local skin damage associated with PEG surgery. The skin around PEG tube is frequently inflamed by gastric juice and chronically irritating skin might induce systemic activation of the immune system, thereby inducing generalized lesions, which might be one of the possible mechanisms. 
PEG is one of the most common endoscopic procedures performed worldwide nowadays, and clinical importance of this procedure has been increased. Therefore, knowing the complications of PEG is very important but none of the studies have demonstrated the relationship between PEG and BP other than ours. Since our study is the retrospective survey conducted at the single institution with small sample size, which does not have strong power. It should be still premature to generalize this finding to other populations. Large-scale clinical prospective studies in multiple medical institutions would be warranted. Since the incidence is much higher among elderly in nursing homes, ${ }^{2}$ and PEG is thought to be performed frequently among these institutionalized elders, the survey should be mainly conducted in nursing homes.

In conclusions, PEG may be a significant risk factor for $\mathrm{BP}$ in some populations. PEG has become the primary choice to establish enteral access for disabled elders unable to take oral feedings lately, the frequency of BP may be increased more in the near future. Since physicians manage these patients with PEG, a greater awareness of this disease is needed for not only dermatologists but also physicians, and they should take leadership in conducting the survey to further confirm the link between BP and PEG, and explore the mechanism.

\section{Disclosure Statement:}

The authors declare that they have no conflicts of interest to disclose. 


\section{References}

99

1001 Schmidt E, Zillikens D. Pemphigoid diseases. Lancet 2013; 381:

$101 \quad 320-332$.

102

103

2 Fernandez-Viadero C, Arce Mateos F, Verduga Velez R, Crespo

104

3 Alpsoy E, Akman-Karakas A, Uzun S. Geographic variations in

4 Bastuji-Garin S, Joly P, Lemordant P, et al. Risk factors for bullous

5 Lo Schiavo A, Ruocco E, Brancaccio G, Caccavale S, Ruocco V, Wolf R.

6 Cecchi R, Paoli S, Giomi A. Peristomal bullous pemphigoid. J Eur

$7 \quad$ Nozu T, Mita H. Bullous pemphigoid and percutaneous endoscopic gastrostomy. Intern Med 2010; 49: 971-975.

8 Downs AM, Lear JT, Bower CP, Kennedy CT. Does influenza vaccination induce bullous pemphigoid? A report of four cases. $B r J$ Dermatol 1998; 138: 363. 